\title{
Poly(thionine)-modified GC Electrode for Simultaneous Detection of Dopamine and Uric Acid in the Presence of Ascorbic Acid
}

\author{
Yong Yuan, A. J. Saleh Ahammad, ${ }^{\dagger}$ Guang-Ri Xu, ${ }^{\ddagger}$ Sunglyuu Kim, ${ }^{\star}$ and Jae-Joon Lee ${ }^{\ddagger}$ \\ Department of Bioscience and Biotechnology' Konkuk University, Seoul 143-701, Korea. 'E-mail: skim100@ikonkuk ac.kr \\ ${ }^{\dagger}$ Department of Applied Chemisty' \& Department of Advanced Technology Fusion, Konkuk University, Chingin 380-701, Korea \\ ${ }^{*}$ E-mall:jlee@ikku.ac.kr \\ ${ }^{\ddagger}$ Henan Institute of Science and Technolog; Ximiang 453003, P.R. Chind \\ Received July: 30, 2008
}

Key Words : Poly(thionine), Simultaneous detection, Dopamine, Ascorbic acid, Electrode nodification

Dopamine (DA) is one of the most significant catecholamines, functioning as a neurotransmitter in the central nervous system and a medicament to drug addiction and Parkinson's disease. ${ }^{12}$ On the other hand, extreme abnormalities of LA levels in the body fluid are symptoms of several diseases, such as gout, hypenuricemia, and LeschNyan." Generally, the electrochemical methods are very simple and advantageous for detection of DA and LA. However, the voltammetric responses of them are not resolved enough, at ordinary electrodes, due to the very close electrochemical redox potentials of both. Another obstacle in monitoring any of them is the interference from ascorbic acid (AA). It usually co-exists with them in the biological fluids at a significantly high concentration range (ca. 0.2-0.4 mM) and it has almost the same redox potential as those of DA and UA.

Therefore, the separation of the electrochemical response of AA, DA, and UA with reasonable sensitivities has been one of the major goal of both electroanalytical and bioelectrochemical research. Various approaches, such as the modification of electrode by polymer film, ${ }^{+}$nano materials, covalent modification, ${ }^{6}$ self-assembled monolayers, ${ }^{7}$ as well as the use of carbon paste electrode ${ }^{8}$ and the electrochemical pretreatment of the electrode ${ }^{y}$ have been developed to distinguish them selectively from each other. The polymermodified electrodes prepared by the electropolymerization have received extensive interests in the detection of analytes because of its enhanced selectivity and sensitivity in addition to the homogeneity and the strong adherence to the electrode surface along with the excellent chemical stability of the $\mathrm{f}_{1} \mathrm{~lm} .^{10}$ In this communication, we present the preliminary results of simultaneous determination of the DA and LA in the presence of AA by using the Poly(thionine) modified glassy carbon electrode (PTGCE).

A series of cyclic voltammograms (CVs) showing the polymerization of the thionine on glassy carbon (GC) was shown in Figure 1. It was obtained by sweeping the potential between -0.4 and $+0.1 \mathrm{~V}$ following the preanodization at $+1.8 \mathrm{~V}$ in $\mathrm{pH} 6.5$ phosphate buffer solution (PBS) containing $1 \mathrm{mM}$ thionine. It shows the monotonic increase of the peak currents of redox waves at a formal potential of ca. $-0.29 \mathrm{~V}$, upon consecutive potential sweep. This is consistent with the results in the literature and clearly indicates the continuous growth of the poly(thionine) film (PTF) on the electrode surface. ${ }^{11}$ The shiny and deep violet-blue colored film is very stable and hard to be taken off from the surface unless the electrode was heavily polished. It is very well known that the polymerization process used in this work generally provides a robust PTF on $\mathrm{GC}$ at near neutral $\mathrm{pH} .{ }^{12}$ However, it has been found that the preanodization process is also required to obtain the stable PTF because the electrochemical activation of $\mathrm{GC}$ is known to induce the large increase in the overall surface roughness (inset of Figure 1). The surface with higher roughness is generally very favorable for adsorption and, consequently, it offers a stronger anchoring of the polymer films onto the electrode surface. In this work, the preanodization at $1.8 \mathrm{~V}$ has been applied in the $\mathrm{pH} 6.5$ PBS containing thionine. The homogeneous distribution of pores, shown in the SEM image of the surface of the GC after the preanodization (Figure 2(a)), is consistent with the formation of the relatively porous surface at the anodization process. The following electrochemical polymerization, during the voltammetric sweep, is expected to occur homogeneously and in a layer-by-layer pattern ${ }^{13}$ to from an uniform coverage of PTF, as indicated in Figure 2(b).

Figure 3 showed the CVs of the mixture of AA, DA, and $\mathrm{UA}(500,20$, and $100 \mu \mathrm{M}$, respectively) in $0.1 \mathrm{MPBS}$ ( $\mathrm{pH}=$

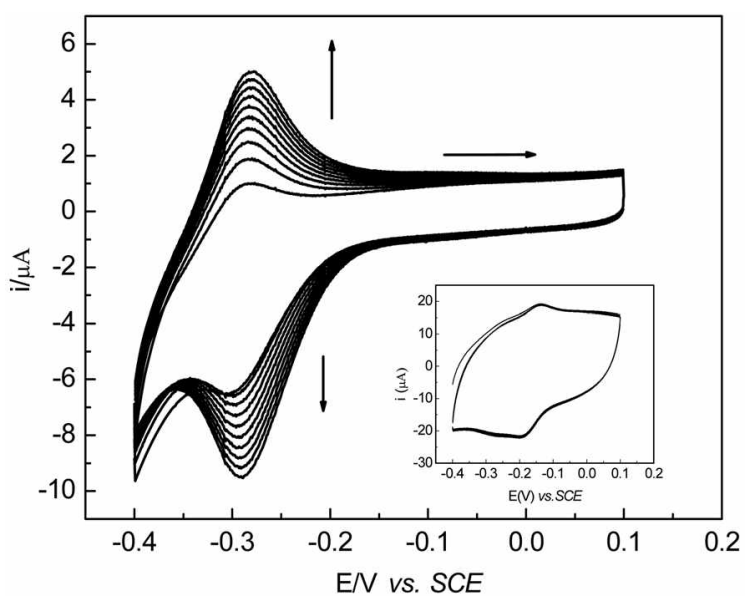

Figure 1. CVs for the electropolymerization of thionine on preanodizd GC in 0.1 M PBS (pH 6.5). The inset shows CV for pure GC. scan rate: $0.1 \mathrm{Vs}^{-1}$. 

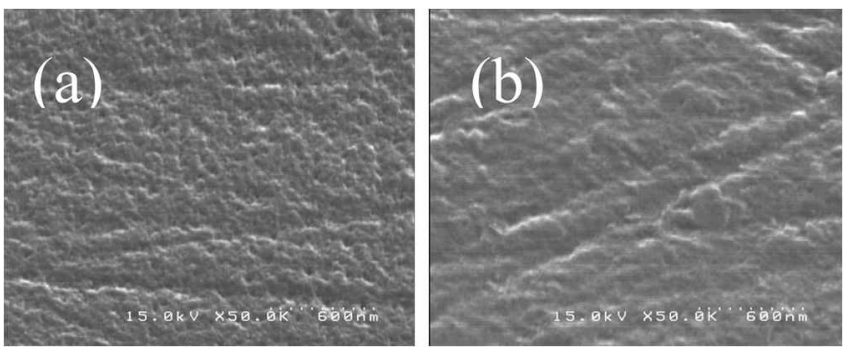

Figure 2. The SEM image of the sufface of the GC after the preanodization (a) and polymerization (b), respectively.

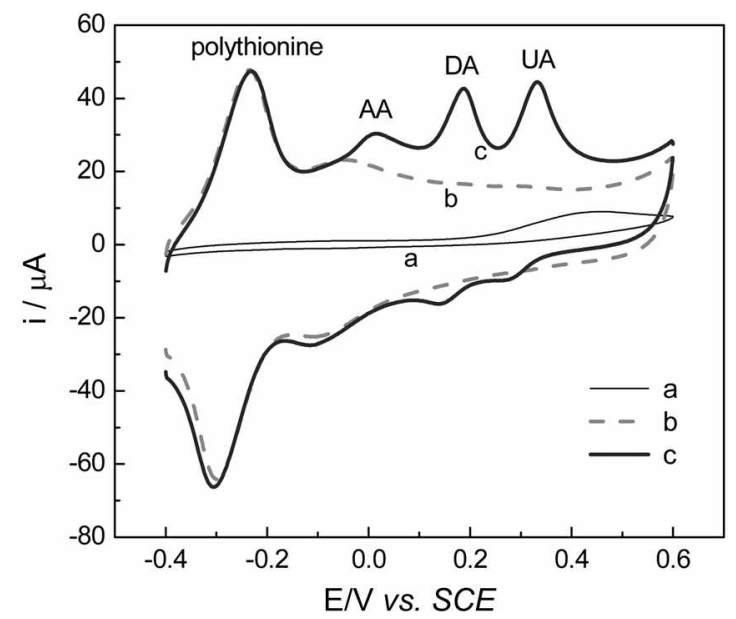

Figure 3. CVs for a mixture of $500 \mu \mathrm{M}$ AA. $20 \mu \mathrm{M}$ DA and 100 $\mu \mathrm{M}$ LA in $\mathrm{pH}$ 6.5 PBS at GCE (a) and PTGCE (c). CV for PTGCE in the blank solution (b).

6.5) at bare GCE and PTGCE. It is obvious that AA. DA. and UA are electrochemically active at both electrodes. However, only a broad single anodic oxidation peak was observed at ca. $0.45 \mathrm{~V}$ for the mixture at the bare GCE (Fig. 3 , a), while the peak potentials for each component were clearly distinguishable at the PTGCE (Fig. 3. c). The curve b is the CV of the PTGCE in 0.1 M PBS with no analyte; It clearly indicates the two oxidations of the PTF at $c a .-0.24$ and $-0.07 \mathrm{~V}$ along with the large background charging current, which implies a significant increase in the capacitance of the electrode upon deposition of the PTF. The appearance of the small oxidation peak observed at $c a$. $-0.07 \mathrm{~V}$ was not clearly resolved in curve $\mathrm{c}$, but it was attributed to the PT from the results of differential pulse voltammetric (DPV) responses.

To verify the applicability of the PTGCE for the selective determination of $\mathrm{DA}, \mathrm{UA}$ and $\mathrm{AA}$, we investigated the current responses of DA and UA in the presence of AA. As shown in Figure 4, both DA and UA showed linear responses, independently, in the concentration range up to ca. $80 \mu \mathrm{M}$. The detection limits for both DA and UA were identified to 1.8 and $1.5 \mu \mathrm{M}$, respectively, according to the conventional definition ( $3 \mathrm{~s})$.

In this communication, we have shown that the PTGCE can resolve the electrochemical response of AA, DA and UA. Further useful applications of this electrode, such as a development of biosensors, are still under investigation by
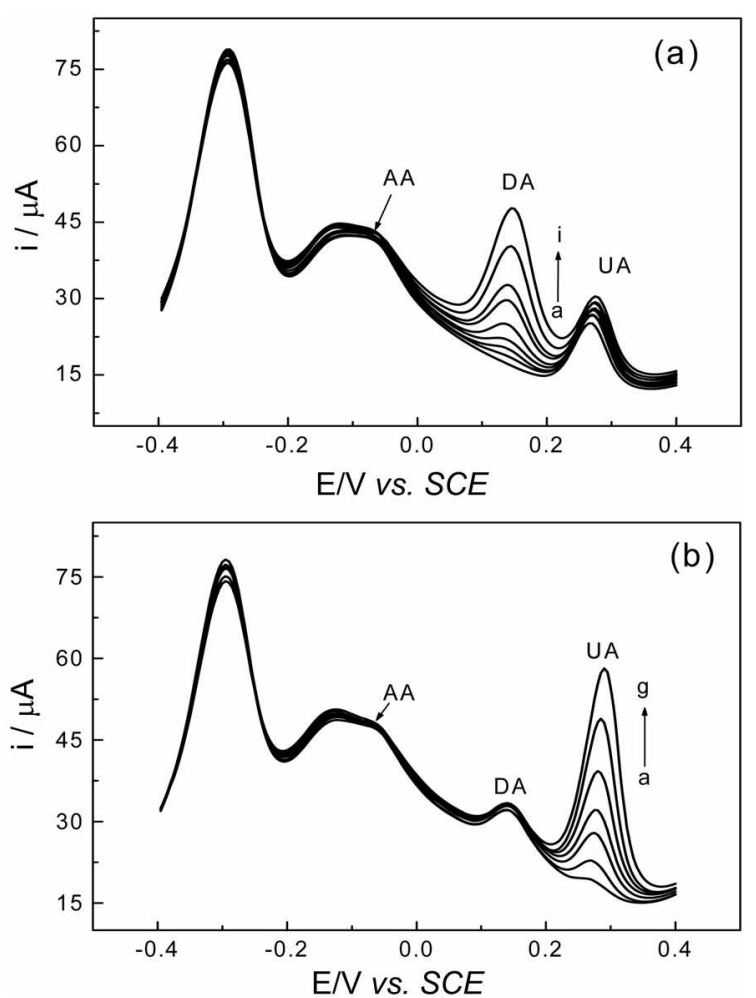

Figure 4, (a) DPV of DA with different concentrations (a-i: 0. 2.4, $6.10 .20,30,50,70 \mu \mathrm{M}$ ) at a PTGCE in the presence of $500 \mu \mathrm{M}$ AA and $15 \mu \mathrm{M}$ LA (b) DPV of LA with different concentrations (a-g: 2, 6, 10, 15. 25, 45, 75, $\mu \mathrm{M}$ ) at PTGCE in the presence of 500 ,$\mu \mathrm{M} \mathrm{AA}$ and $15, \mu \mathrm{M} \mathrm{DA}$.

taking advantage of the ability of the selective detection of various catecholamine-derivatives and similar neurotransmitters.

Acknowledgments. This work was supported by the faculty research fund of Konkuk University in 2004.

\section{References}

1. Chang, H.-Y.; Xu, G.-R.; Park, Y.; Lee, K. P.; Bate, Z.-LI. Butl. Korean Chem. Soc. 2004, 35,1261 .

2. Park, S.-K.; Lee, N.-S.; Lee, S.-H. Bull. Korean Chem. Soc. 2000, 21,959

3. Dutt, V. V. S. E.; Mottola, H. A. Anol. Chem. 1974, 46, 1777.

4. Selvaraju, T.; Ramaraj, R. J. Appl. Electrochem. 2003, 33, 759.

5. Bae, S. R.; Jeong, H.; Jo, S.; Jeon, S. Bull. Korean Chem. Soc. 2007, 28, 2363

6. Li, Y.; Lin, X. Sens. Actwator B-Chen. 2006, 115, 134.

7. Rai, C. R.; Tokuda, K.; Ohsaka, T. Bioelectrochemistry 2001, 53, 183 .

8. Zare, H. R.; Nasirizadel, N.; Ardakani, M. J. Electroanal. Chem. 2005, 577, 25

9. Premkumar, J.; Khoo, S. B. J. Electroanal. Chem. 2005, 576, 105

10. Bernd, K.; Zagorska, M. Sinth. Met. 1989, 33, 257.

11. Yang, R.; Ruan, C.; Dai, W.; Deng, J.; Kong, J. Electrochim. Acta $1999,44,1585$

12. Xu, Y.; Jiang, Y.; Yang, L.; He, P. G.; Fang, Y. Z. Chin. J. Chem. $2005,23,1665$

13. Liu, C.; Lu, G.; Jiang, L.; Jiang, L.; Zhou, X. Electroanalysis 2006, 18,291 